This is the peer reviewed version of the following article: Gajebasia, S. , Pearce, J. , Redman, M. , Johnson, M. and Finn, G. (2019), How can training in care of the dying be improved?. Clin Teach, which has been published in final form at

https://doi.org/10.1111/tct.12999. This article may be used for non-commercial purposes in accordance with Wiley Terms and

Conditions for Use of Self-Archived Versions.

\title{
How can training in care of the dying be improved?
}

\section{Gajebasia, Sareena; Pearce, Jessica; Redman, Melody; Johnson, Miriam; Finn, Gabrielle}

\section{Abstract: Background}

Care of the dying patient is an intrinsic part of the role of foundation year foctors (FYs). This study aimed to explore FYs' experiences of training and their perceived training needs for their role in care of the dying.

Methods

All FYs in one foundation school were invited to take part in semi-structured group or individual interviews. Eight group interviews and 21 individual interviews were conducted with a total of 47 participants. Interview recordings were transcribed verbatim and framework analysis was undertaken.

Findings

Key themes derived from the interviews included FYs teaching opportunities regarding care of the dying and their learning methods for this subject matter which included learning from experience, observation, simulation, written guidance and supervision. Areas for further training was another key theme and training needs identified included prescribing, communication, recognising dying, documentation, societal perspective and emotional resilience.

Discussion

FYs' training experiences in this area vary. This study identifies training needs which can be used to inform both undergraduate and postgraduate curricula.

\section{Introduction}


This is the peer reviewed version of the following article: Gajebasia, S. , Pearce, J. , Redman, M. , Johnson, M. and Finn, G. (2019), How can training in care of the dying be improved?. Clin Teach, which has been published in final form at

https://doi.org/10.1111/tct.12999. This article may be used for non-commercial purposes in accordance with Wiley Terms and Conditions for Use of Self-Archived Versions.

In the UK, following medical school graduation, foundation year doctors (FYs)

undertake a two-year training programme within a foundation school that oversees their training. FYs are expected to care for dying patients and the General Medical Council mandates that UK graduates will be able to do this ${ }^{1}$. However, undergraduate education has been criticised for failing to prepare doctors for care of the dying ${ }^{2}$. FYs feel unprepared $^{3}$ and have unfulfilled learning needs ${ }^{4}$.

UK guidance on care of the dying was published in 'Priorities for Care of the Dying Person'5. Five areas of care are recommended: 'recognise'; 'communicate'; 'involve'; 'support'; and 'plan and do'5. The aim of this study was to explore FYs' experiences in care of the dying. We posed three research questions (table 1). We found that FYs' experiences are variable and, within the five priorities, ${ }^{5}$, good practice and areas for improvement exist ${ }^{6}$. In this paper we present the primary data analysis related to question three.

[Table 1]

\section{Methods}

We conduced a qualitative study using semi-structured face-to-face, telephone and videoconferencing interviews to allow for exploration of experiences and to maximise participation. All FYs in the North Yorkshire and East Coast foundation school in England were invited through email, social media, posters and word-of-mouth to form a 
This is the peer reviewed version of the following article: Gajebasia, S. , Pearce, J. , Redman, M. , Johnson, M. and Finn, G. (2019), How can training in care of the dying be improved?. Clin Teach, which has been published in final form at

https://doi.org/10.1111/tct.12999. This article may be used for non-commercial purposes in accordance with Wiley Terms and

Conditions for Use of Self-Archived Versions.

convenience sample. Participants provided informed consent and completed a

demographic questionnaire.

'Priorities for Care of the Dying Person'5 was used as the conceptual framework because it outlines nationally recognised areas for care. The conceptual framework was used to derive the standardised interview question stems alongside other published literature. Following a pilot interview, which was formed of one videoconferencing group interview with three participants, the questions stems (table 2) were agreed.

[Table 2]

Between January and March 2016, we held eight group interviews (two to five participants each) and twenty-one individual interviews, each conducted by one researcher (JP/MR/SG/GF). Interviews were recorded and transcribed verbatim. Due to the use of a conceptual framework, data were analysed in accordance with framework analysis $^{7}$, with familiarisation of the interview transcripts, line by line coding, formation of a coding framework, charting of the data and interpretation. There were some predetermined codes as a result of the conceptual framework and interview questions but the authors were open to other codes as the data were inductively analysed. Coding was primarily conducted by MR but reviewed by others. The themes and sub-themes were formed from the pre-determined codes and codes found on analysis. Data analysis software was used to organise the data. Thematic saturation was reached as no new 
This is the peer reviewed version of the following article: Gajebasia, S. , Pearce, J. , Redman, M. , Johnson, M. and Finn, G. (2019), How can training in care of the dying be improved?. Clin Teach, which has been published in final form at

https://doi.org/10.1111/tct.12999. This article may be used for non-commercial purposes in accordance with Wiley Terms and Conditions for Use of Self-Archived Versions.

themes emerged from the data. Ethical approval was granted by Hull York Medical

School; institutional approvals were granted from involved NHS trusts.

\section{Findings}

Forty-seven (14\%) out of 335 FYs participated and none withdrew. Forty-four of the participants had attended medical schools in the United Kingdom (UK).

\section{Themes}

Three main themes emerged: 1) learning opportunities, 2) varied methods for learning and 3) self-identified training needs (table 3).

\section{[Table 3]}

For anonymity, the data are identified by interview type (group (G), individual (I)), interview number and, where relevant, participant number $(\mathrm{P})$.

\section{Theme 1. Learning opportunities}

The teaching received varied.

"I found my medical school was really good at teaching end of life care."(G8P4)

"I haven't had any end of life care training..."(I4)

Timeliness of teaching was important. 
This is the peer reviewed version of the following article: Gajebasia, S. , Pearce, J. , Redman, M. , Johnson, M. and Finn, G. (2019), How can training in care of the dying be improved?. Clin Teach, which has been published in final form at

https://doi.org/10.1111/tct.12999. This article may be used for non-commercial purposes in accordance with Wiley Terms and Conditions for Use of Self-Archived Versions.

“...we had like breaking bad news communicating skill sessions but I know that they're quite early on, like the first clinical year [of medical school], when you maybe don't have enough actual medical knowledge to really appreciate them properly. "(G7P4)

Theme 2. Varied methods for learning

A. Learning from experience. Participants valued experience, particularly with practical skills.

"I think it's very much...experienced based, like the more time to talk about and the more people and family talk to, you're a bit more prepared each time..."(G4P1)

B. Learning from observation. Learning from observation was recognised as beneficial.

“...we actually had one week of placement in fourth year and final year in a palliative hospice, which I found was very useful again because you see the dayto-day management, erm, of a palliative patient...in real time, it's not something you're going to read in, in a book or something you're going to learn ...on a theoretical basis..."(G3P2)

C. Simulation. The value of simulation was noted.

“...it's a good way to kind of practice that for real life." (G8P1)

D. Written guidance. Written guidance was a helpful resource. 
This is the peer reviewed version of the following article: Gajebasia, S. , Pearce, J. , Redman, M. , Johnson, M. and Finn, G. (2019), How can training in care of the dying be improved?. Clin Teach, which has been published in final form at

https://doi.org/10.1111/tct.12999. This article may be used for non-commercial purposes in accordance with Wiley Terms and Conditions for Use of Self-Archived Versions.

"This is the [end of life care] booklet with the drugs, fantastic, and it's a really useful guide..."(G8P4)

E. Supervision. Supervision from senior colleagues, nursing staff and palliative care specialists was useful.

“...he [palliative care consultant] would always take the time to explain to me, you know, why are we doing this?"(G3P2)

Theme 3. Self-identified training needs

A. Prescribing. Participants highlighted a lack of confidence with a desire for guidance.

"I felt very unprepared when I had to prescribe it [anticipatory medications] for the first time...."(G1P3)

"I think a lot more guidance on palliative [care] and what kind of medications you can give...would be really useful." (G2P1)

B. Communication. Communication skills were identified as a learning need.

"I've had situations where relatives have asked, 'Well, by giving my Mum this medication, are you not erm, bringing her death closer... actually that's one of the kind of questions that we need training on how to answer."(G1P3)

C. Recognising dying. Participants would value training on recognising dying.

"Yes, training...would have been helpful, it's sort of recognising a patient who is dying..."(I6) 
This is the peer reviewed version of the following article: Gajebasia, S. , Pearce, J. , Redman, M. , Johnson, M. and Finn, G. (2019), How can training in care of the dying be improved?. Clin Teach, which has been published in final form at

https://doi.org/10.1111/tct.12999. This article may be used for non-commercial purposes in accordance with Wiley Terms and Conditions for Use of Self-Archived Versions.

D. Documentation. Training needs on how to document in the clinical record, complete death certificates and cremation forms were identified.

“...it's not something we're, we're really taught either, like I never remember having tutorials on like how to document like end of life care."(I9)

E. Societal perspective. Participants commented on their lack of knowledge about the legal and ethical factors involved and their understanding of different cultural beliefs with regards to dying.

“...I don't know where the...legal or ethical boundaries come... with regards to end of life." (G3P2)

“...people coming in with different religions and from different countries...they see death in different ways and they want things done in certain ways..."(I1)

F. Emotional resilience. Death presented emotional challenges and preparation for this was lacking.

“... a really important aspect is, erm, how to emotionally deal with...the patient dying, but it's something that...was never taught or discussed in medical school."(I13)

\section{Discussion}

Given FYs are expected to care for dying patients, it is important to understand their training experiences and needs. The variation in training, despite curriculum specification, was notable. Training needs ranging from biomedical aspects of clinical practice through to psychosocial issues and personal resilience were identified. The 
This is the peer reviewed version of the following article: Gajebasia, S. , Pearce, J. , Redman, M. , Johnson, M. and Finn, G. (2019), How can training in care of the dying be improved?. Clin Teach, which has been published in final form at

https://doi.org/10.1111/tct.12999. This article may be used for non-commercial purposes in accordance with Wiley Terms and Conditions for Use of Self-Archived Versions.

clinical and psychosocial training needs map to the foundation programme curriculum item of 'manages palliative and end of life care' ${ }^{8}$. Addressing these learning needs will also enable FYs to be able to achieve the 'Priorities for Care of the Dying Person's recommendations.

There is a need to improve training and ensure learning opportunities are maximised for undergraduates and postgraduates, for example, the use of hospices as training sites ${ }^{9}$. Consistent with previous findings ${ }^{2,3,10}$ our participants described learning about care of the dying through experience, observation, simulation and written and verbal guidance, therefore these methods should be further utilised to meet FYs' needs. It was noted that the timing of this learning had significant impact on its value and that it should occur when it was most clinically relevant. Our data showed that exposure to care of the dying was welcomed as a medical student, particularly during more senior years, and that simulation exercises in a safe environment had a valuable role. In practice, spiral curriculum approaches would seem appropriate, where earlier "safer" practice could be built on when the topic was revisited later in the course after more clinical exposure. This education could be further supported by training early in the foundation programme. Within this spiral curriculum, emotional resilience could be fostered with each learning event. Table 4 outlines recommendations for training. 
This is the peer reviewed version of the following article: Gajebasia, S. , Pearce, J. , Redman, M. , Johnson, M. and Finn, G. (2019), How can training in care of the dying be improved?. Clin Teach, which has been published in final form at

https://doi.org/10.1111/tct.12999. This article may be used for non-commercial purposes in accordance with Wiley Terms and

Conditions for Use of Self-Archived Versions.

Strengths of this work include reaching thematic saturation and using a convenience

sample, which may have resulted in recruiting participants with extreme views.

Limitations include relying on participants' memory of events, over months/years, and participants having a variable post duration at the time of the interviews (between five and 19 months) which may have resulted in different opportunities.

MR, JP and SG, were FYs at the time of conducting the interviews, which may have helped participants express their opinions, or it may have been a barrier to discussions due to fear of peer judgement. To ensure fair data interpretation GF and MJ, both experienced researchers and not FYs, were involved in data analysis.

\section{Conclusions}

Training experiences vary and training needs exist around prescribing, communication, recognising dying, documentation, societal perspective and emotional resilience. Many of these training needs reflect the foundation programme syllabus and when addressed will help FYs achieve the 'Priorities for Care of the Dying Person'5 recommendations. It is important to acknowledge the FYs' expressed training needs and use them to improve training. Teaching this topic at clinically relevant times in a spiral curriculum is suggested as a way to deliver this teaching across undergraduate and early postgraduate training using existing learning methods.

\section{References}

1. General Medical Council. Outcomes for graduates (Tomorrow's Doctors). General Medical Council [Internet]. 2015 [cited 2018 September 3]. Available 
This is the peer reviewed version of the following article: Gajebasia, S. , Pearce, J. , Redman, M. , Johnson, M. and Finn, G. (2019), How can training in care of the dying be improved?. Clin Teach, which has been published in final form at

https://doi.org/10.1111/tct.12999. This article may be used for non-commercial purposes in accordance with Wiley Terms and Conditions for Use of Self-Archived Versions.

from https://www.gmc-uk.org/-/media/documents/outcomes-for-graduates-jul-

$$
\text { 15-1216_pdf-61408029.pdf }
$$

2. Gibbins J, McCoubrie R, Forbes K. Why are newly qualified doctors unprepared to care for patients at the end of life? Med Educ. 2011;45(4):389-399.

3. Bowden J, Dempsey K, Boyd K, Fallon M, Murray SA. Are newly qualified doctors prepared to provide supportive and end-of-life care? A survey of foundation year 1 doctors and consultants. J R Coll Physicians Edinb. 2013;43(1):24-28.

4. Linklater GT. Educational needs of foundation doctors caring for dying patients. J R Coll Physicians Edinb. 2010;40(1):13-18.

5. Leadership Alliance for the Care of Dying People. Priorities for care for the dying person: duties and responsibilities of health and care staff. Leadership Alliance for the Care of Dying People. 2014 [cited 2017 October 22]. Available from http://webarchive.nationalarchives.gov.uk/20160506190405/http://www.nhsiq.n hs.uk/media/2485900/duties_and_responsibilities_of_health_and_care_staff__with_prompts_for_practice.pdf.

6. Redman M, Pearce J, Gajebasia S, Johnson M, Finn G. Care of the dying: a qualitative exploration of foundation year doctors' experiences. Med Educ. 2017;51(10):1025-1036.

7. Gale NK, Heath G, Cameron E, Rashid S, Redwood S. Using the framework method for the analysis of qualitative data in multi-disciplinary health research. BMC Med Res Methodol. 2013;13:117. 
This is the peer reviewed version of the following article: Gajebasia, S. , Pearce, J. , Redman, M. , Johnson, M. and Finn, G. (2019), How can training in care of the dying be improved?. Clin Teach, which has been published in final form at

https://doi.org/10.1111/tct.12999. This article may be used for non-commercial purposes in accordance with Wiley Terms and Conditions for Use of Self-Archived Versions.

8. UK foundation programme office. Syllabus. UK foundation programme office [Internet]. 2016 [cited 2017 October 22]. Available from:

http://www.foundationprogramme.nhs.uk/curriculum/Syllabus.

9. Arolker M, Barnes J, Gadoud A, Jones L, Barnes L, Johnson MJ. “They’ve got to learn" - a qualitative study exploring the views of patients and staff regarding medical student teaching in a hospice. Palliat Med. 2010; 24(4):419-426.

10. Parikh PP, Brown R, White M, Markert RJ, Eustace R, Tchorz K. Simulationbased end-of-life care training during surgical clerkship: assessment of skills and perceptions. J Surg Res. 2015;196(2):258-263.

Table 1

Research Questions 
This is the peer reviewed version of the following article: Gajebasia, S. , Pearce, J. , Redman, M. , Johnson, M. and Finn, G. (2019), How can training in care of the dying be improved?. Clin Teach, which has been published in final form at

https://doi.org/10.1111/tct.12999. This article may be used for non-commercial purposes in accordance with Wiley Terms and Conditions for Use of Self-Archived Versions.

1) What are the experiences of FYs?

2) How do these relate to the five 'Priorities for Care of the Dying Person'5?

3) How can any findings inform under- and post-graduate medical education curricula?

Table 2

\section{Question Stems}


This is the peer reviewed version of the following article: Gajebasia, S. , Pearce, J. , Redman, M. , Johnson, M. and Finn, G. (2019), How can training in care of the dying be improved?. Clin Teach, which has been published in final form at

https://doi.org/10.1111/tct.12999. This article may be used for non-commercial purposes in accordance with Wiley Terms and Conditions for Use of Self-Archived Versions.

What training have you had and how well has it prepared you?

Have you had any training in the 5 priorities (as outlined in the Priorities for Care of the Dying Person ${ }^{5}$ ) and how could this be improved?

Are there any specific areas you would like training on? What would be the best way to provide this? What else could be done to better prepare you?

Table 3

\begin{tabular}{|l|l|}
\hline Themes & 1. Learning opportunities
\end{tabular}


This is the peer reviewed version of the following article: Gajebasia, S. , Pearce, J. , Redman, M. , Johnson, M. and Finn, G. (2019), How can training in care of the dying be improved?. Clin Teach, which has been published in final form at

https://doi.org/10.1111/tct.12999. This article may be used for non-commercial purposes in accordance with Wiley Terms and Conditions for Use of Self-Archived Versions.

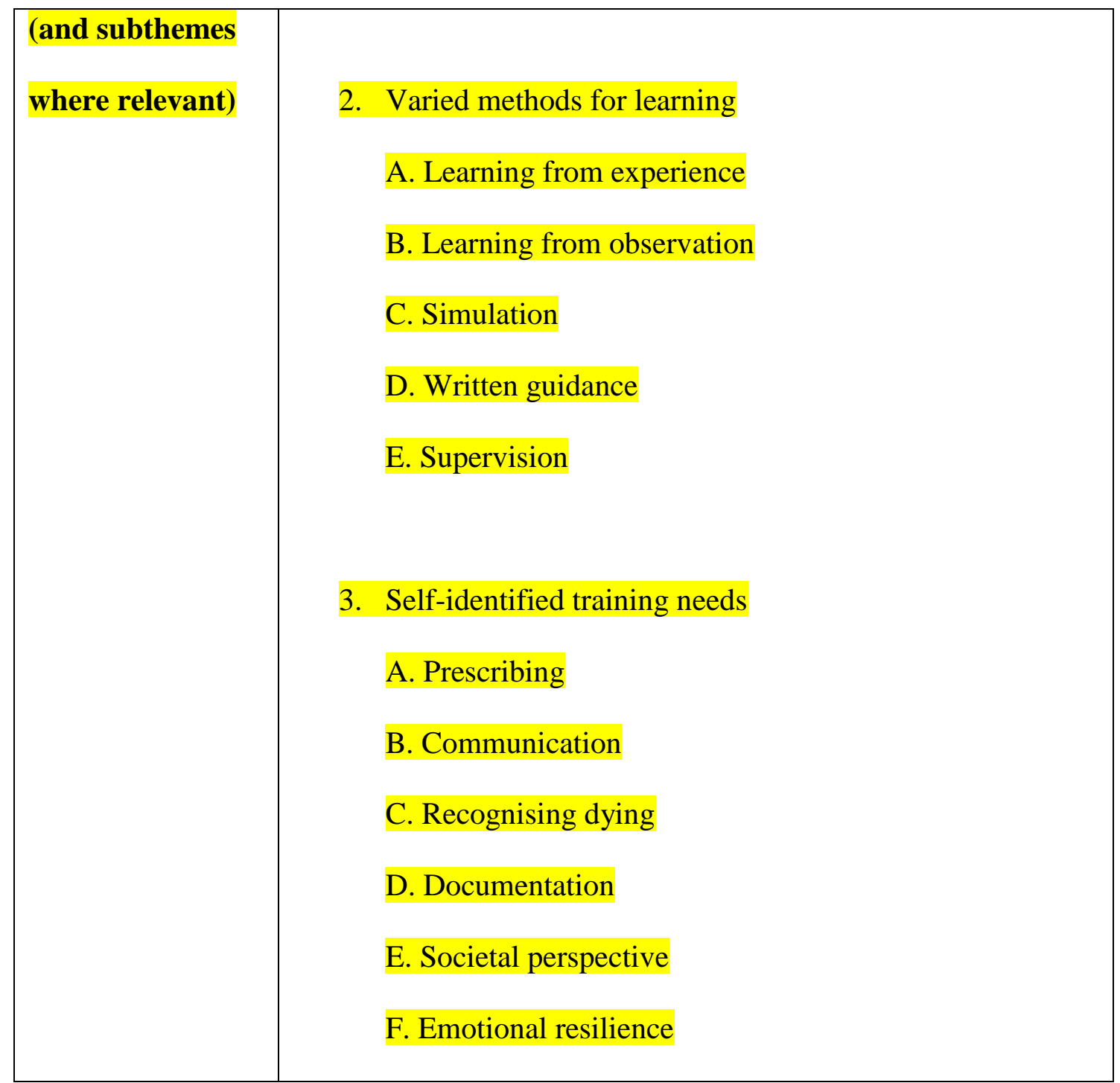

Table 4 
This is the peer reviewed version of the following article: Gajebasia, S. , Pearce, J. , Redman, M. , Johnson, M. and Finn, G. (2019), How can training in care of the dying be improved?. Clin Teach, which has been published in final form at

https://doi.org/10.1111/tct.12999. This article may be used for non-commercial purposes in accordance with Wiley Terms and

Conditions for Use of Self-Archived Versions.

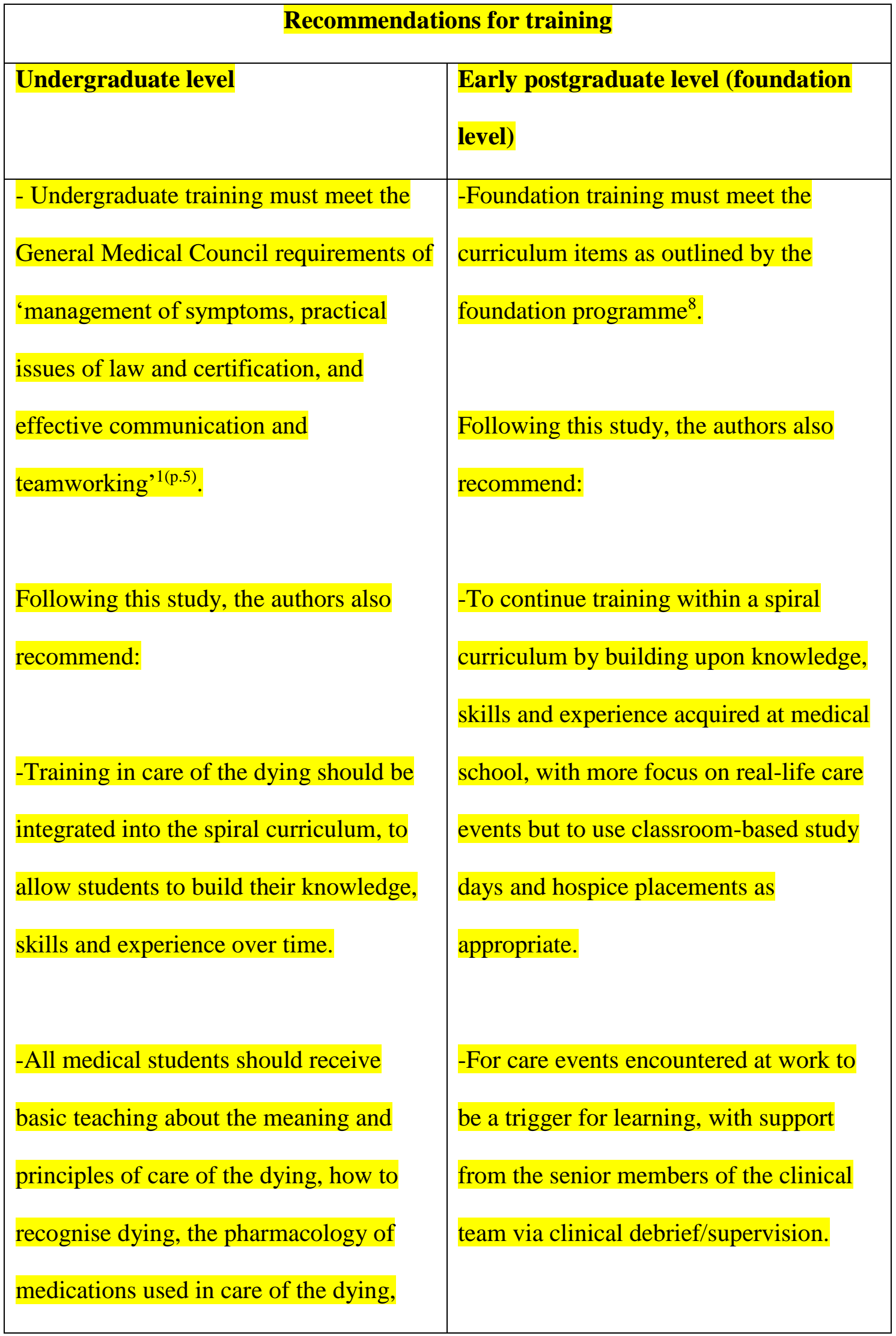


This is the peer reviewed version of the following article: Gajebasia, S. , Pearce, J. , Redman, M. , Johnson, M. and Finn, G. (2019), How can training in care of the dying be improved?. Clin Teach, which has been published in final form at https://doi.org/10.1111/tct.12999. This article may be used for non-commercial purposes in accordance with Wiley Terms and Conditions for Use of Self-Archived Versions.

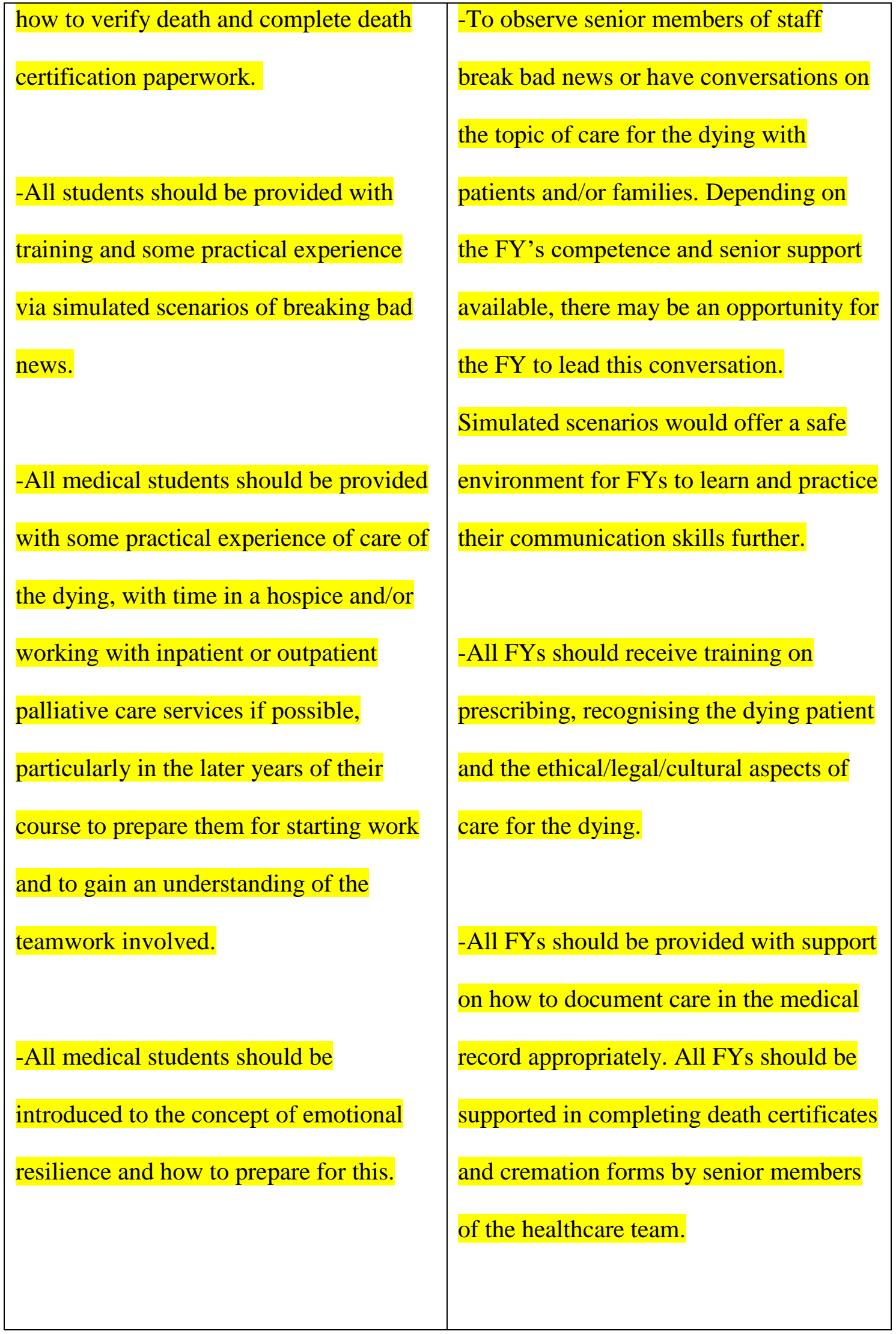


This is the peer reviewed version of the following article: Gajebasia, S. , Pearce, J. , Redman, M. , Johnson, M. and Finn, G. (2019), How can training in care of the dying be improved?. Clin Teach, which has been published in final form at

https://doi.org/10.1111/tct.12999. This article may be used for non-commercial purposes in accordance with Wiley Terms and Conditions for Use of Self-Archived Versions.

-All FYs should be made aware of where
NHS Trust guidance on care of the dying
can be accessed and how to contact the
palliative care team.
-All learning events or care of the dying
experiences should be an opportunity for
building emotional resilience, with
support from more senior members of the
healthcare team.


This is the peer reviewed version of the following article: Gajebasia, S. , Pearce, J. , Redman, M. , Johnson, M. and Finn, G. (2019), How can training in care of the dying be improved?. Clin Teach, which has been published in final form at

https://doi.org/10.1111/tct.12999. This article may be used for non-commercial purposes in accordance with Wiley Terms and Conditions for Use of Self-Archived Versions.

Dr. Sareena Gajebasia (SG), Dr. Jessica Pearce (JP) and Dr. Melody Redman (MR), with supervision from Dr. Gabrielle Finn (GF) and Professor Miriam Johnson (MJ), undertook this work in partial fulfilment of the academic foundation programme training in the North Yorkshire and East Coast foundation school.

SG: developed the concept and contributed to the study's protocol. Involved in data collection and data interpretation. Prepared the first draft of the manuscript and contributed to revisions of the intellectual content.

JP: developed the concept and contributed to the study's protocol. Involved in data collection and data interpretation. Contributed to the intellectual content of the paper. MR: developed the concept and contributed to the study's protocol. Involved in data collection and data interpretation. Contributed to the intellectual content of the paper.

GF: Contributed to the study's protocol. Involved in data collection and data interpretation. Contributed to the intellectual content of the paper.

MJ: Contributed to the study's protocol. Involved in data interpretation. Contributed to the intellectual content of the paper. 